Sabine Maasen

\title{
Innovation und Relevanz: Forschung im Gerangel widerstreitender Anforderungen
}

\begin{abstract}
In science policy, current concepts like "Responsible Research and Innovation" or "Citizen Science" document a new understanding of the relationship between science and society: Beyond being innovative, science should also engender an entirely new responsive, participatory, and sustainable research culture. Simultaneously, civil society has been demanding a responsible science as well as citizen participation for some time. These expectations manifest in increasing evaluations, selective research funding and demands for relevance and usefulness, producing new research formats. By way of two instructive examples - the Human Brain Project and Citizen Science -, this contribution asks whether we are on our way to a new 'contract' between science and society, in which science has to be legitimated through innovations that are characterised by excellence and relevance. My conclusion is: Excellence and relevance cannot be readily reconciled, but they are related to each other through pragmatic compromises (managerialisation, public engagement).
\end{abstract}

Zusammenfassung: Aktuelle wissenschaftspolitische Konzepte wie „Responsible Research and Innovation“ und „Citizen Science“ dokumentieren ein neues Verständnis der Beziehung von Wissenschaft und Gesellschaft: Wissenschaft soll nicht nur innovativ sein, sondern es soll eine neue responsive, partizipative und nachhaltige Forschungskultur entstehen. Parallel dazu fordert seit einiger Zeit auch die Zivilgesellschaft eine verantwortliche Wissenschaft sowie Teilhabe von Bürgerinnen und Bürgern. Diese Erwartungen schlagen sich in zunehmenden Evaluationen, selektiver Forschungsförderung und Nützlichkeitsanforderungen nieder und bringen neue Formate der Forschung hervor. Dieser Beitrag geht anhand zweier instruktiver Beispiele, dem Human Brain Projekt und der Cititzen Science, der Frage nach, ob wir uns derzeit auf dem Weg zu einem neuen ,Vertrag“ zwischen Wissenschaft und Gesellschaft befinden, in dem Wissenschaft sich durch Innovation im Modus von Exzellenz und Relevanz legitimieren muss. Das Fazit lautet: Exzellenz und Relevanz lassen sich nicht umstandslos versöhnen, sondern werden in pragmatischen Kompromissen (Managerialisierung, soziale Öffnung) aufeinander bezogen.

\section{Innovationen und Innovationen der Innovationen}

Dass das Wort ,Sprunginnovation“ es (noch) nicht zum Unwort des Jahres gebracht hat, mag daran liegen, dass wir allenthalben und dermaßen von Innovationen umstellt sind - technischen, ökonomischen und sozialen -, dass wir sie, mehr denn je, ohnehin für die wichtigste Grundlage gesellschaftlicher Wohlfahrt und diesen 
Betriebsmodus gesellschaftlicher Reproduktion für schlichtweg evident halten - allen Aufrufen zu mehr Nachhaltigkeit ${ }^{1}$ und Degrowth (Burkhart/Schmelzer/Treu 2017) zum Trotz. Interessanter noch: Es tragen sich auch die letztgenannten Konzepte unterdessen immer umstandloser in die Innovationssematik ein, wie es Ideen „ökologischer Innovation“ (Fussler 1996; Schiederig/Tietze/Herstatt 2012; bzw. soziologisch Blättel-Mink 2001) oder ,innovation for degrowth“ (Pansera/Owen 2018) bezeugen. Innovationstätigkeit erreicht jedoch nicht nur immer mehr Handlungsbereiche der Gesellschaft; sie tut dies auch immer schneller. Schließlich sorgen digitale Infrastrukturen dafür, dass wir Disruptionen zwar durchaus nicht überall erleben, aber zumindest überall erwarten - nicht zuletzt dank einer aufgeregten Medialisierung (Weingart 2005; Weingart et al. 2017) und erheblicher wissenschaftspolitischer Förderanstrengungen. ${ }^{2}$

All dies lässt die Wissenschaft nicht unberührt. Der Goldstandard der Forschung, so scheint es, liegt immer weniger in der Grundlagenforschung: Ihr wird ohne Unterlass elfenbeinerne Selbstgenügsamkeit und $\mathrm{zu}$ geringe Beachtung gesellschaftlicher Problemlagen attestiert. Es ist vor allem Innovationstätigkeit, die wissenschaftliche Forschung heute überzeugend legitimiert. Sie ist es, die auf scheinbar zwanglose Weise akademische curiositas mit gesellschaftlicher Relevanz verbindet. Innovationstätigkeit sichert die Legitimation für Forschung und die erheblichen Investitionen in sie. Der Umkehrschluss scheint indessen nicht länger möglich. Zwar benötigt Innovativität ohne Frage den Bezug auf wissenschaftlich hergestelltes, daher verlässliches Wissen. Das allein aber reicht immer seltener.

Dass die Verbindung von Forschung und Innovation durchaus nicht einfach ist, zeigt indessen schon der Umstand, dass Innovationen Gesellschaften nicht einfach voranbringen, sondern erst einmal irritieren. Innovationen unterbrechen immerhin Gewissheiten, Handlungsroutinen sowie Deutungsmuster, und in Innovationsgesellschaften geschieht dies immer wieder: meist ungleichzeitig und überwiegend unkoordiniert; Innovationen transzendieren das Bekannte. Das sollen sie ja auch, aber das macht Arbeit. Gesellschaften müssen mit technischen, ökonomischen, sozialen, politischen, ökologischen und weiteren Innovationen zurechtkommen, sich auf sie einstellen, sie ablehnen, umdeuten oder subvertieren, nicht selten eingedenk bekannter oder unbekannter Risiken, die man teils billigend in Kauf nimmt, teils argwöhnisch trotzdem nutzt. Atomkraft, Stammzellforschung, Nanomaterialien, Robotik, Informatik, Automatisierung der Mobilität ... - wohin man auch immer schaut, spätestens seit den 1960er Jahren wird Kritik etwa an psychischen oder physischen, ethischen oder politischen, ökologischen oder ökonomischen Implikationen der Forschung und ihrer technologischen Manifestationen laut.

1 https://www.bundesregierung.de/breg-de/themen/nachhaltigkeitspolitik/die-deutsche-nachhaltigkeitsstrategie-318846 [30.09.2019].

2 https://www.bundesregierung.de/breg-de/themen/digital-made-in-de/foerderung-digitaler-technologien-und-innovationen-1546800 [30.09.2019]; https://ec.europa.eu/digital-single-market/en/ pillars-digitising-european-industry-initiative [30.09.2019]. 
Die immer enger werdende Kopplung von Forschung an Innovation geschieht seither vor allem über die Frage der Relevanz: Wissenschafts- und Technologieentwicklung sollen nicht nur innovativ, sondern auch nachhaltig, partizipativ und neuerdings verantwortlich geschehen. Technikfolgenabschätzung (,Technology Assessment': TA; Bröchler/Simonis/Sundermann 1999), ,Public Understanding of Science“ (PUS; Irwin/Wynne 1996; Gregory/Lock 2008), ,Public Engagement in Science and Technology“ (PEST; Hagendijk/Irwin 2006), ,Ethical, Legal and Social Implications of Science‘ (ELSI; Felt/Fochler/Strassnig 2011; Yesley 2008), verstärkte Wissenschaftskommunikation (Bucchi/Trench 2008; Chilvers 2013) und ,Citizen Science‘ (Haklay 2013; Dickel/Franzen 2016) widmen sich dem Ziel, unerwünschte Nebenfolgen wissenschaftlich-technischer Entwicklungen frühzeitig zu erkennen. Offenbar erfüllen alle die genannten Programme die Aufgabe einer Verbindung von Forschung, Innovation und Relevanz nur unzureichend. Wohl deshalb erscheinen immer neue, und sie überbieten einander: zeitlich, sachlich und sozial. In zeitlicher Hinsicht gilt es, nicht erst nachträglich an den unerwünschten Folgen einer Innovation zu laborieren, sondern so frühzeitig wie nur möglich über mögliche Folgen nachzudenken; in sachlicher Hinsicht reicht es nicht aus, die Öffentlichkeit nur zu informieren, sie muss sich auch effektiv mit den Forschenden austauschen können; in sozialer Hinsicht sind Bürgerinnen und Bürger nicht länger nur als Adressatinnen und Adressaten von Wissenschaft anzusprechen: Warum können sie nicht auch mitforschen?

Es sind kumulierte Ansprüche wie diese, die in jüngerer Zeit eine nochmals neue Programmatik hervorgebracht haben, um Innovationen für relevante Anspruchsgruppen relevant zu gestalten: Seit einigen Jahren hören wir nun von ,Responsible Research and Innovation' (RRI; Owen/Macnaghten/Stilgoe 2012). Wissenschaftliche Entwicklungen sollen nicht länger nur nachträglich bewertet (downstream) oder vorab visioniert (upstream) werden, sondern der Forschungsprozess selbst soll - midstream - im Hinblick auf den verantwortungsbewussten Umgang mit Innovationen begleitet werden. Mit den Dimensionen Antizipation, Reflexivität, Inklusivität und Responsivität umfasst RRI auch Ethik, Gendergerechtigkeit und Wissenschaftskommunikation. Im Zuge dessen erweitert sich der Geltungsbereich für Reflexivität und Verantwortlichkeit der Forschung: Er betrifft, zumindest im Prinzip, die wissenschaftliche Praxis aller Disziplinen, Grundlagen- ebenso wie Anwendungsforschung; er gilt immer dauerhafter und er involviert immer mehr Anspruchsgruppen. Insbesondere (aber nicht nur) in Forschungsdomänen nahe an Mensch und Gesellschaft wie der Medizin, aber auch etwa in der Umweltforschung, Mobilitätsentwicklung oder Stadtplanung vereinen Projekte, die RRI-Prinzipen folgen, nicht nur Forscherinnen und Forscher verschiedener Disziplinen (Interdisziplinarität), sondern auch Patientinnen und Patienten oder Bürgerinnen und Bürger als Mitforschende sowie professionelle Akteure beispielsweise aus dem Krankenhaus, der Forstwirtschaft oder der Stadtplanung (Transdisziplinarität), und dies fallweise unter Einbezug ethischer oder genderbezogener Fragen. Überdies widmen sie sich der Wissenschaftskommunikation im Sinne eines Public Engagement, das nicht nur die Information der Bürgerinnen und 
Bürger, sondern auch die Interaktion zwischen Expertinnen und Experten aus der Wissenschaft und dem jeweiligen Einsatzfeld (hier etwa der Klinik, dem Klimaprojekt oder dem Stadtquartier) vorsieht. Sie wirkt idealiter auf die Forschung zurück.

Innerhalb weniger Jahre avancierte die Idee von ,Responsible Research and Innovation' und ihre Abkürzung RRI zu einem prominenten Thema im Programm „Horizon 2020“ der Europäischen Kommission ebenso wie ihrer Konferenzen (unter der dänischen EU-Ratspräsidentschaft 2012) und wissenschaftlichen Publikationen ${ }^{3}$ (neben Owen 2012 etwa Grunwald 2011 oder Hahn/Ladikas 2014). Nota bene: Bereits vor 15 Jahren machte sich ein Diskurs über verantwortungsvolle Entwicklung der Nanotechnologie bemerkbar. Seither ist er allerdings nicht nur in der Wissenschaft, in Forschungseinrichtungen und Institutionen der Forschungsförderung aufgestiegen, sondern auch in Unternehmen sowie in der Politik. Er hat überdies bereits zu weiteren spezifischen Programmen wie etwa ,Corporate Social Responsibility“ oder ,Social Entrepreneurship‘ sowie zu konkreten Maßnahmen (Foresight, Dialogprogramme etc.) geführt. Zumindest programmatisch zieht dies entscheidende Weichenstellungen hinsichtlich Innovation und Verantwortung nach sich: Es entsteht soeben eine neue Architektur verteilter Wissenserzeugung, aber auch geteilter Verantwortung.

Die Innovation von Innovation, die sich hier geltend macht, ist eine zweifache. Zum einen erreicht die Forderung nach gesellschaftlich relevanter Innovationstätigkeit unter dem Stichwort ,Responsibility“ den Forschungsprozess selbst: von der Fragestellung, über das interdisziplinären Design bis zur engagierenden Kommunikation auch über den engeren Fachdiskurs hinaus soll sie durch Wissenschaftlichkeit und Relevanz bestimmt sein. Zum anderen mag man sich erinnern, dass noch in den 1990er Jahren im Innovationsdiskurs ,Exzellenz‘ und ,Transdisziplinarität‘ als zwei distinkte Qualitätsdiskurse mit je eigenen Praktiken der Qualitätssicherung auftraten (Maasen 2008). Während die Einen auf Exzellenz setzten, favorisierten die Anderen Relevanz. Mit dem Konzept ,Verantwortlicher Forschung und Innovation“ sollen nun aber solche Wissensproduktionen epistemische und gesellschaftliche Autorität genießen, denen es gelingt, beides: Exzellenz und Relevanz zu überbrücken.

Man ahnt, dass mit dieser doppelten Innovation der Innovationstätigkeit viel versprochen wird, was indessen nicht umstandslos einzulösen ist: immerhin sind immer weitreichendere Projektionen zukünftiger Anwendungs- und Problemlösungsmöglichkeiten, die erhebliche Investitionen öffentlicher Fördermittel binden, schon weit vor ihrer Einlösung zu begründen. RRI muss dazu die ohnehin mit vielfältigen Unsicherheiten behafteten Übersetzungsschritte in der Forschung vom Agenda-Setting bis zur Implementierung einer Reihe von deliberativen und mitforschenden Interventionen zugänglich machen, die Kommunikation und Koordination erfordern. Und ist

3 https://ec.europa.eu/programmes/horizon2020/en/h2020-section/responsible-research-innovation [30.09.2019]; https://ec.europa.eu/research/index.cfm?pg=events\&eventcode=82DACA6D-9025-C419-7CE417A83303B1C0 [30.09.2019]. 
das dann noch Wissenschaft, gar bessere Wissenschaft, wie es die Idee der Demokratisierung der Wissenschaft nahelegt (Maasen 2010)?

Die folgende Studie wird die gegenwärtige Prominenz von RRI zunächst als Dynamik sich wandelnder Verhältnisse (oder ,Verträge') zwischen Wissenschaft und Gesellschaft rekonstruieren und dann der Frage nachgehen, worin der Neuigkeitswert von RRI besteht. Die Antwort: Legitimation durch Innovation (qua verlässlichem Wissen) wird durch Maßnahmen der Legitimation für Innovation (qua Relevanz) ergänzt. Sodann zeigt sich, dass auch verantwortliche Forschung an systematische Grenzen ihrer praktischen Erfüllung gerät: Am Fall des ,Human Brain Projects‘ lässt sich verdeutlichen, wie verantwortliche Forschungs- und Innovationstätigkeit durch Managerialisierung handhabbar gemacht wird: Ethics-Management ist auf sachlicher Ebene die Antwort auf schier uneinlösbare inter- und transdisziplinäre Kommunikations- und Koordinationserfordernisse. Am Fall der Beteiligung von Bürgerinnen und Bürgern an Forschung im Modus der ,Citizen Science‘ lässt sich weiterhin exemplarisch zeigen, dass sich hier Wissenschaft zwar sozial, nicht aber epistemisch öffnet.

Der Beitrag endet mit einem Räsonnement über den aktuellen Stand des Gesellschaftsvertrags mit der Wissenschaft. Forschung befindet sich derzeit im Gerangel widerstreitender Anforderung - Innovation! und Relevanz! Nicht nur werden Maßnahmen der Relevanzerzeugung im Innovationsprozess durch epistemische Kriterien der Forschungstätigkeit erheblich eingeschränkt und haben nicht selten eher ostentativen Charakter, sondern darüber hinaus darf nicht vergessen werden, dass die Forderung nach Relevanz mit einer anderen Forderung nach wie vor konkurriert: derjenigen nach Exzellenz. Exzellenz erhöht die Chance auf innerwissenschaftliche und Relevanz die Chance auf gesellschaftliche Anerkennung. Beide Ziele miteinander zu versöhnen, so das Fazit, ist zwar wissenschaftspolitisches Programm, stellt jedoch die Wissenschaft besonders in anwendungsnahen Feldern häufig vor Probleme. Nurpragmatische Lösungen rufen regelmäßig Kritik und alsbald neue Programme hervor.

\section{Immer neue Verträge zwischen Wissenschaft und Gesellschaft ...}

Was rezent unter dem Label RRI auftritt, kann als Teil eines bereits länger andauernden Diskurses verstanden werden, in dem die Suche nach einem neuen ,Vertrag“ (Guston 2000) zwischen Wissenschaft und Gesellschaft zum Ausdruck kommt. Der Begriff des ,Vertrags‘ ist ausdrücklich als Metapher zu verstehen: Denn obwohl er nirgendwo kodifiziert ist, umfassen die Verhältnisse von Wissenschaft und Gesellschaft doch stets eine ganze Reihe von „Arrangements von Vertrauen und Kontrolle, die in verschieden Ländern und zu verschiedenen Zeiten getroffen worden sind, und unter denen die Wissenschaft gefördert wird“ (Weingart 2011: 46). 
Die moderne Wissenschaft als Methode und Praxis der systematischen und verlässlichen Wissensproduktion hat sich seit ihrem Beginn durch ein epistemisches Ethos leiten lassen, mit dem sie sich selbst reguliert: Gute wissenschaftliche Praxis und gute Wissenschaftlerinnen und Wissenschaftler bemühen sich um Widerspruchsfreiheit, Kohärenz, Klarheit, Sparsamkeit (das lex parsimoniae bzw. Ockham’s Razor), Genauigkeit und Überprüfbarkeit. Diese Prinzipien kritischer Selbstkontrolle sichern zugleich die Autonomie der Wissenschaft und die Unabhängigkeit der Wissenschaftlerinnen und Wissenschaftler. In den 1940er Jahren fasst Robert Merton dieses wissenschaftliche Ethos in den vier bekannten CUDOS-Prinzipien zusammen: Kommunitarismus, Universalismus, Interesselosigkeit und Organisierter Skeptizismus. Die ausdauernde Kritik an diesen Prinzipien und ihre Ergänzung sind hier weniger bedeutsam als das Spezifikum des Arrangements von Vertrauen und Kontrolle: „Das ,scientific ethos‘ ist durch normative (und sozialwissenschaftlich zugängliche) institutionalisierte Imperative und Sanktionen, Begründungs- und Verfahrensregeln, Belohnungs- und Kritiksysteme konstituiert, die die Praxis der Wissenschaft und das Selbstverständnis der Wissenschaftsgemeinschaft anleiten“ (Özmen 2015: 67) - es ist dieses Gefüge von Normen und Verfahren der verantwortlichen Selbstkontrolle von Peers, in das die Gesellschaft vertraut.

In den 1970er Jahren rücken mit der Frage der Verantwortung der Wissenschaft für ihre risikoreichen technischen Anwendungen zunächst die Individualtugenden der einzelnen Wissenschaftlerpersönlichkeit in den Vordergrund. Sie sollen die epistemische Integrität und Qualität der Wissenschaft sichern: Objektivität, Ehrlichkeit, Toleranz, disziplinierte Skepsis und selbstlose Hingabe treten zu dem gemeinschaftlichen Ethos der ,Scientific Community ‘ hinzu:

The (scientific) code should explicitly take cognizance of the fact that the scientist is an individual who lives in a society which has ends other than the cognitive ends of scientists, and that the cognitive achievements of scientists do not always and necessarily serve these ends. (Cournand/ Zuckerman 1970)

Dieses neue Arrangement von Freiheit und Kontrolle bekräftigt Art. 5 Abs. 3 des Grundgesetzes: Die Freiheit von Wissenschaft, Forschung und Lehre sind als ein defensives und konstitutives Individualrecht ohne Gesetzesvorbehalt konzipiert. Einschränkungen können nur durch eine Kollision mit gleichwertigen Rechtsgütern wie Würde, Leben, körperliche Unversehrtheit begründet werden. Zugleich wird jedoch auf eine andere Ebene der Regulierung und Kontrolle verwiesen, nämlich die Verantwortung der einzelnen Wissenschaftlerpersönlichkeit und der Wissenschaftsgemeinschaft.

Interne Verantwortung trägt der Wissenschaftler gegenüber den Regeln guter wissenschaftlicher Praxis. Externe Verantwortung im Sinne der Rechenschaftspflicht für die möglichen Anwendungen und Folgen seiner Forschung ist hingegen kein konstitutiver Bestandteil des epistemischen Ethos der Wissenschaft oder der Integritätsnormen des einzelnen Wissenschaftlers, sondern eine genuin ethische Norm. (Özmen 2015: 69) 
Der sich soeben abzeichnende Gesellschaftsvertrag setzt indessen weniger auf Vertrauen in die einzelnen Wissenschaftlerinnen und Wissenschaftler sowie die Wissenschaftsgemeinschaft, sondern zunehmend auf Prozeduren der Kontrolle. Eine kurze Rekapitulation idealtypischer Gesellschaftsverträge mit der Wissenschaft seit 1945 soll diesen Wandel verdeutlichen:

- Das lineare Modell: Der implizite Vertrag zwischen Wissenschaft und Gesellschaft nach dem Zweiten Weltkrieg war noch durch das Leitbild einer losen Kopplung von Politik und Wissenschaft geprägt. Die Aufgabe der Wissenschaft wurde darin gesehen, nach eigenen Regeln Grundlagenwissen zu produzieren, das ultimativ in nützliche Technologien übersetzbar sein sollte. Die Rolle des Gemeinwesens bestand hingegen darin, die Wissenschaft für diese Aufgabe mit Ressourcen auszustatten, nicht aber in deren Selbstregulation einzugreifen. Dieser am ,linearen Innovationsmodell' (Godin 2016) orientierte Vertrag zwischen Wissenschaft und Gesellschaft war zutiefst vom Vertrauen in die Eigenrationalität und die verantwortliche Selbststeuerungskapazität des Wissenschaftssystems geprägt (Guston 2000). Debatten um mögliche externe Zweckbestimmungen wissenschaftlicher Forschung stießen hingegen sowohl in der Wissenschaft selbst als auch im politischen System weithin auf Ablehnung (Lax 2015: Kapitel 1.1).

- Das finalisierte Modell der 1970er Jahre richtet sich auf die zentrale Idee, dass Disziplinen sowohl vor als auch nach ihrer zunehmenden theoretischen Schließung die Richtung der Theorieentwicklung nicht allein determinieren. Insbesondere über die theoretische Relevanz weiterer Forschung muss daher nach zusätzlichen Kriterien entschieden werden. In diesen Phasen der Wissenschaftsproduktion liegen die Voraussetzungen dafür, dass eine von wissenschaftlich-technischen Interessen (Innovation!) und sozialen Bedürfnissen (Relevanz!) gleichermaßen getragene Forschungsplanung möglich ist (Böhme/van den Daele/Krohn 1973). Nach Vorstellung der Autoren sollte die sogenannte Finalisierungsthese zu einer ,wissenschaftstheoretischen Kartographie‘ ausgearbeitet und der Wissenschaftspolitik für eine rationale Forschungsplanung an die Hand gegeben werden (Wieland 2009: 14-15).

- Das hybridisierte Modell, das sich seit den 1990er Jahren herausbildet, macht auf einen nochmals neuen Modus der reflexiven Wissensproduktion (,Mode 2“) aufmerksam (Gibbons et al. 1994). In allen seinen idealtypischen Charakteristika scheint es auf eine stärkere Verflechtung von Wissenschaft und der übrigen Gesellschaft hinzuweisen: Anwendungsorientierte Wissensproduktion bezieht Bedarfe potentieller Anwender bereits in die Formulierung der Problemstellung ein; Transdiziplinarität sichert die Überwindung disziplinärer Grenzen; neben Hochschulen werden auch außeruniversitäre Forschungseinrichtungen bis hin zu Beratungsunternehmen und ,think tanks' in die Wissensproduktion einbezogen; die zunehmende Verschränkung von Forschungs- und Anwendungskontexten sensibilisiert die Forschenden für die gesellschaftlichen, wirtschaftlichen und politischen Folgen ihres Wissens und ihrer Technologien (,Social Accoun- 
tability'); die Qualitätskontrolle durch Peers wird durch außerwissenschaftliche Kriterien ergänzt.

Im Kontrast zu seinen Vorgängermodellen forciert das hybridisierte Modell weder eine radikale (lineares Modell) noch eine begrenzte Autonomie der Wissenschaft (finalisiertes Modell). Stattdessen favorisiert es programmatisch eine kontinuierliche und umfassende Berücksichtigung externer Ansprüche. Geltung und Reichweite dieses Modells werden bis heute kritisch diskutiert (ein frühes Beispiel: Weingart 1997) - niemand bestreitet jedoch ernstlich, dass Ansprüche an die Berücksichtigung außerepistemischer Ansprüche gewachsen sind und immer neue Versuche aus sich hervortreiben, die gesellschaftliche Erwartung sowohl an die ,Innovativität durch Forschung ' als auch an die ,Legitimität der Forschung' miteinander in ein produktives Verhältnis zu bringen.

\section{RRI: Nach den Verträgen oder auf dem Weg zu einem neuen Vertrag?}

Was genau bedeutet eine derart gesteigerte Berücksichtigung externer Ansprüche? In dem 2001 erschienenen Buch des Autorenkollektivs um Helga Nowotny, das der Präzisierung dieser These um Mode 2 einer erneuerten Form der Wissenschaftsproduktion dienen sollte, kreist ein wesentliches Argument um die Versicherung, dass es im Diesseits sich beständig ausweitender externer Ansprüche doch einen „epistemischen Kern der Wissenschaft“ gebe, der sich nicht transwissenschaftlich verhandeln lasse (Nowotny/Scott/Gibbons 2001). Diese Spannung zwischen epistemischem Kern und gesteigerter Externalisierung der Wissenschaft wird seither mit immer neuen normativen und/oder epistemischen Überlegungen, Programmatiken und Prozeduren aufzulösen versucht. Doch: Die stets zunehmenden gesellschaftlichen Ansprüche sowohl an die ,Innovativität durch Forschung‘ als auch an die ,Legitimität der Forschung' sind offensichtlich nicht umstandslos zu überbrücken. Innovatives Forschungshandeln betritt per definitionem wissenschaftliches Neuland mit unvollständigem Wissen um seine politischen oder sozialen Folgen; in dem Maße, in dem Innovation zur Signatur der Gegenwartsgesellschaft wird (Rammert et al. 2016), steigern und vervielfältigen sich zugleich die Wahrnehmung von Risiko, Nichtwissen und Ungewissheit - es mehren sich mithin die Fragen, wie man trotzdem handeln soll, individuell und kollektiv (Böschen/Schneider/Lerf 2004). Verantwortliche Innovation scheint die Antwort auf diese Fragen zu sein.

Die Ergänzung des wissenschaftsinternen epistemischen Ethos um eine wissenschaftsexterne Ethik der Verantwortung ist jedoch ebenfalls nicht neu: Sie wird seit der Beteiligung von Wissenschaftlerinnen und Wissenschaftler an der Entwicklung von militärischen Massenvernichtungswaffen (dem Manhattan-Projekt) diskutiert. Ab 
den 1960er Jahren nimmt diese Forderung in Verbindung mit der ökologischen Kritik des technologischen Imperativs eine systematische wissenschaftsethische Form an. Hierzu bieten moderne Lebenswissenschaften, Nano-, Informations- und Kommunikationswissenschaften weitere Anlässe; auch die verschwimmenden Grenzen von Grundlagen- und Anwendungsforschung plausibilisieren den Ruf nach verantwortlicher Wissenschaft um ein Weiteres.

Wissenschaft und Wissenschaftspolitik antworten darauf erstens mit einer Kaskade von reflexiven Programmatiken: Technikfolgenabschätzung (TA), Public Understanding of Science (PUS), Public Engagement in Science and Technology (PEST), Ethical Legal and Social Implications of Science (ELSI), verstärkte Wissenschaftskommunikation etc. Zweitens reagieren sie mit einer Vermehrung von Instrumenten der Externalisierung von Kontrolle wie Ethik-Kodizes der wissenschaftlichen Fachgesellschaften und vielfältige Evaluationen als Governance-Instrumente (Knie/ Simon 2008). Drittens transformiert sich Wissenschaftsproduktion selbst: Förderinstrumente verlangen vermehrt nach Responsivität, Nachhaltigkeit und Partizipation der Forschung selbst (Maasen/Dickel 2016). Auf diese Weise bildet sich ein reflexives Innovationsregime heraus (Lindner et al. 2016: 67ff.): Es verwebt antizipatorische, integrative und experimentelle Kapazitäten in ein Ensemble von problembezogenen, situativ zu kombinierenden reflexiven Prinzipien, Beteiligungs-Verfahren und Controlling-Instrumenten.

In dieser Hinsicht kann RRI auch als eine „soziale Innovation“ (Föger et al. 2016) gelten: Es sind weniger die generellen Ziele (,social robustness of knowledge') noch die Dimensionen, Instrumente und Controlling-Mechanismen selbst, die seine Neuheit ausmachen; es ist ihre forschungsbegleitende Nutzung und ihr Effekt der laufenden Adjustierung von Forschungs- und Innovationstätigkeit. Sie soll nun zentraler Bestandteil von Wissenschaft und Technologieentwicklung selbst werden. Verantwortung ist nun nicht mehr allein eine Sache der Individualtugend von Forscherinnen und Forscher oder ein Normengefüge in der Gemeinschaft der Wissenschaftlerinnen und Wissenschaftler. Gefordert ist nun eine neue moralische Arbeitsteilung, die durch ein ganzes Netzwerk von Forschenden und weiteren am Forschungsprozess beteiligten Akteuren getragen ist.

Was nach einem Versöhnungsprogramm von Innovation und Verantwortung klingt, stößt jedoch mit Blick auf ersten Praktiken bereits auf Kritik. Ein Zwischenfazit des Büros für Technikfolgen-Abschätzung beim Deutschen Bundestag (TAB) vom Dezember 2016 (Lindner et al. 2016a) hält fest: Zwar wird eingefordert, über das Handeln in Alternativen nachzudenken, die Spannungen, Konflikte und Auseinandersetzungen über epistemische, normative (ethische, politische, rechtliche) sowie nicht zuletzt ökonomische Fragen bleiben hingegen bislang weitgehend ausgeblendet. Auch lägen Überlegungen zur konkreten Ausgestaltung des Umgangs mit Alternativenproduktion bisher kaum vor. Wie können die verschiedenen Ansätze, Verfahren und Methoden, die zu einer ,reflexiven Innovationsgestaltung' beitragen sollen, sinnvoll miteinander verknüpft werden? 
Das Collingridge-Dilemma (Collingridge 1982) besagt: Je weiter entwickelt eine Technik ist, desto sicherer kann man die Folgen abschätzen. Allerdings besteht dann keine Möglichkeit mehr, die Technik oder die Technikfolgen gestaltend zu beeinflussen, denn dann ist die Entwicklung entweder bereits abgeschlossen oder bereits so weit fortgeschritten, dass ein Umsteuern kaum noch oder nicht mehr möglich ist gleich, durch welchen Akteur. Und umgekehrt: Je weniger entwickelt eine Technik ist, desto weniger kann man die Folgen abschätzen und konkretes Steuern wird schwierig bis unmöglich. Durch die erhöhte Responsivität, die TA, PUS, PEST oder ELSA herstellen, macht sich dieses Dilemma nun auch im Forschungs- und Innovationsprozess selbst sowie in weiteren involvierten Akteursgruppen bemerkbar, es demokratisiert sich. Alle fragen sich, mit jeweils unterschiedlichen Antworten: Wann ist der richtige Zeitpunkt zum Mitgestalten gekommen? Einig sind sich Viele, dass man sich am Anfang gemeinsam über Themen, Fragen und Ziele der Forschung verständigen, während des Forschungsprozesses immer wieder den Stand der Dinge überprüfen und ggf. korrigieren sollte (nicht zuletzt mit Blick auf die vielfältigen Implikationen ihres möglichen Ergebnisses). Ein laufendes Monitoring der Forschungsergebnisse und ihrer Wirkungen schließt die „Relevanzschöpfungskette“ ab. Doch jenseits solch allgemeiner Rezepturen wird dann viel über konkrete Umsetzungen und wechselseitige Überforderungen gestritten. Nicht zuletzt gilt RRI, wie seine Vorgängerprogramme, vor allem für exzellenzgetriebene Innovationsforschung tendenziell als ,Bremse‘.

Das Dilemma verschärft sich mithin: RRI nährt einerseits den Bedarf nach immer neuen und wirkungsvolleren Kommunikations- und Beteiligungsverfahren; andererseits ist ein ganz spezifischer Umgang mit diesem erhöhten Responsivitätsanspruch aufgrund des Innovations- und Erfolgsdrucks in hochkompetitiver Forschungs- und Innovationstätigkeit zu erwarten. Bekanntlich lassen sich Dilemmata auch nicht lösen, man kann sie nur immer wieder angehen. Aus dieser Perspektive erscheint RRI als ein weiterer Ansatz, neue Regeln, neue Prozeduren und neue ,gute‘ Praktiken zu artikulieren, die wissenschaftlich und gesellschaftlich genügen, zugleich effizient sind und innovationsfördernd - nur, dass man nicht vorher festlegen kann, was ,gut sein wird und empirisch überdies oft das Unerwartete passiert. Die Erwartungen an Innovation durch Forschung und Legitimation für Innovation werden immer höher. Neben die Überbietungsdynamik immer neuer, Relevanz sichernder Programmatiken gesellen sich praktische Coping-Strategien, von denen hier zwei exemplarisch illustriert werden sollen: Zum einen die Managerialisierung komplexer sozioepistemischer Koordinationsanforderungen in einem Big Science Projekt (3.1); zum andern die soziale, kaum aber epistemische Öffnung der Forschungs- und Innovationstätigkeit in Citizen Science Projekten (3.2). 


\subsection{Das Human Brain Project: Managerialisierung erhöhter sozio- epistemischer Koordinationsanforderungen}

Das Verbundvorhaben Human Brain Project (HBP) ist ein neuro- und informationstechnologisches Infrastrukturprojekt im europäischen Maßstab. Zentral sind neuartige Forschungsplattformen, auf denen alle Daten und Kenntnisse, die über die Struktur und Funktion des Gehirns bekannt sind, zusammengeführt und dazu verwendet werden, integrierte Gehirnmodelle zu erstellen, die durch Simulationen auf Supercomputern validiert werden können. Neben 10 Forschungsprojekten etwa zu Brain Simulation oder Neurorobotics und einem Koordinationsprojekt ist die Aufgabe des Teilprojekts 12, ethische, soziale und philosophische Aspekte der Forschung im HBP frühzeitig zu erkennen und dazu beizutragen, dass sie offen adressiert werden. Die Frage ist nun, ob die problembezogene und prozessbegleitende Justierung reflexiver Governance neuroinformatischer Infrastrukturinnovationen überhaupt gelingen kann, wenn „Ethics and Society“ in ,work packages“ adressiert werden, die erstens eher auf Kompetenzsteigerung der Forschenden (Kommunikation und Dialog) als auf Kollaboration mit Stakeholdern setzen, und zweitens überwiegend auf Entwicklung von Prinzipien und Standards des Ethikmanagements im HBP statt auf die Deliberation und Mitgestaltung techno-sozialer Fragen ausgerichtet sind. Die folgenden Beobachtungen stützen sich auf Angaben auf der Website des HBP, ${ }^{4}$ insbesondere zu Teilprojekt 12.

Während das „Foresight Lab“ an der frühzeitigen Erstellung von Szenarien zu ethisch relevanten Aspekten insbesondere in den Feldern ,Future Neuroscience‘, ,Future Medicine‘, ,Future Computing arbeitet und dies in Reports und Publikationen dokumentiert, befasst sich das ,work package „Neuroethics and Philosophy““ mit philosophischen, ethischen und sozialen Analysen zu Kernaktivitäten und -themen des HBP. Beide Arbeitspakete sollen einerseits die Forschung der Neuro- und Informationswissenschaften konzeptionell anreichern und andererseits die reflexiven Kompetenzen der HBP-Forschenden erhöhen. Beides wird ausdrücklich als ,capacity building، der Forschenden im HBP adressiert. Auch das Arbeitspaket „Public Dialogue and Engagement" steht weniger im Zeichen von kollaborativer Praxis als von Dialog mit öffentlichen und privaten Stakeholdern sowie der Öffentlichkeit. Es versteht sich als Frühwarnsystem in Bezug auf heraufziehende Kontroversen, gibt Empfehlungen an die Forschung und formuliert Forschungsprioritäten. Diesem Vorgehen gelingt es zwar, gesellschaftliche Implikationen des neuroinformatischen Infrastrukturprojekts mit Hilfe von Sozial- und Geisteswissenschaftlerinnen und -wissenschaftlern fallweise und hoch sichtbar zu thematisieren. In diesem Großforschungsverbund geht es jedoch weniger um tatsächliche Interaktionen mit der Gesellschaft: Stattdessen wird erstens „Gesellschaft“ überwiegend von Experten imaginiert und zweitens werden Forschende für Kommunikation mit der überwiegend imaginierten Gesellschaft trai-

4 https://www.humanbrainproject.eu [30.09.2019]. 
niert - die Begegnung mit Gesellschaft geschieht durch (und beschränkt sich auf) Reports, populäre Publikationen und dosierten Dialog.

Das Teilprojekt „Ethics and Society“ verwendet mithin, so bezeichnet es selbst sein Programm, einen erheblichen Teil seiner Kapazität auf „Ethics Management“. Als Teil der Unternehmensethik richtet sich Ethics-Management darauf aus, zielgerichtet, systematisch und aufeinander abgestimmt verbindliche moralische Handlungsmaßstäbe in alle unternehmerischen Entscheidungsprozesse einzubinden. Die in diesem Arbeitspaket $\mathrm{zu}$ entwickelnden Maßnahmen zur ethischen Governance der HBP kreisen um „compliance, reflection, and engagement with ethics among the entire HBP“. Der Import der Unternehmensethik in die Umgebung von Großforschung und Innovation im Rahmen einer EU-Bürokratie ist deutlich: Ethisch relevante Aktivitäten des HBP werden gesammelt, dokumentiert, und für alle Teilprojekte zur Verfügung gestellt. „This ensures that ethical issues are managed to the highest standards within the HBP and develops international good practice.“

Die Formatierung von RRI als Ethics Management umfasst nicht weniger als

- die Einrichtung eines eigenen Arbeitspakets

- Aufgaben im Feld von Standardisierung und deren Dokumentation

- ein umfangreiches Berichtswesen

- Formulierung von Empfehlungen

- genuine, spezialisierte geistes- und sozialwissenschaftliche Forschung

- laufende interne und externe Vernetzungsarbeit zur Sicherung guter wissenschaftlicher Praxis;

das sind die zentralen institutionellen Zurichtungen von RRI im HBP; darüber hinaus gibt es ein Advisory Board und FAQs. Dies zeigt: Dem drängenden Aufruf zur verantwortungsbewussten Forschung wird zwar mit dem Ausbau wohlorganisierter institutioneller Strukturen und Prozesse und auch dafür eingerichteter Positionen begegnet und so die Komplexität anspruchsvoller sozio-epistemischer Koordinationsleistungen reduziert. Die Integration heterogener Interessen und Werte in die wissenschaftliche Praxis und etwa die Anpassung der Agenda bleibt allerdings fraglich. Im Format des add-on zu den zehn Forschungspaketen im Zentrum des Verbundvorhabens ist, so scheint es, den wissenschaftspolitischen Forderungen der EU in Sachen Responsivität Rechnung getragen, ohne jedoch die Forschungspraxis selbst durchgreifend zu ändern.

\subsection{Citizen Science: Soziale Öffnung: ja - epistemische Öffnung: eher nein}

Citizen Science will nichtwissenschaftliche Akteurinnen und Akteure realiter in die Forschung integrieren. So soll die fachwissenschaftliche Perspektive inhaltlich ergänzt und das derart produzierte Wissen sozial robuster und politisch einflussreicher werden. Dies stellt eine erhebliche Herausforderung für die Wissenschaft dar: Im 
Zuge seiner zunehmenden Institutionalisierung und Ausdifferenzierung seit dem ausgehenden 19. Jahrhundert haben nur Personen mit einer zertifizierten akademischen Ausbildung und beruflich-institutionellen Einbindung Zugang zu wissenschaftlicher Forschung. Die Annahme ist, dass Forschung gerade wegen ihrer sozialen Schließung objektives, reliables und valides Wissen erzeuge; nur so sei sie vor äußerer Einflussnahme (etwa spezifischen Interessen der Industrie) geschützt und gewähre Evidenz und Exzellenz (Dickel 2017).

Genau dies steht seit einiger Zeit infrage: Wissenschaftliche Erkenntnisse gelten dann als sozial robuster, wenn sie durch den Einschluss gesellschaftlicher Wissensbestände zustande kommen (vgl. Nowotny et al. 2001: 209ff.). Auf den Startseiten der Homepages einiger Citizen Science Projekte (CSP) wird dies bereits im Slogan deutlich: „Empowering citizen scienctists to invent medicine“ (EteRNA) Geschichte, unser Archiv“ (Topothek) ${ }^{6}$. Auch im Fall des CSP „Patient Science“7 wird betont, dass Betroffene „maximal beteiligt“ werden, um Alltagsprobleme von Mukoviszidose-Erkrankten zu erforschen. In all diesen Fällen wird die Idee der Beteiligung an das Versprechen geknüpft, auf diese Weise das Wissen der Forschenden zu ergänzen oder zu verändern.

Tatsächlich werden auf aufwändige Weise durchaus weitreichende Mitwirkungschancen hergestellt, die allerdings nicht zum Kern der wissenschaftlichen Wissensproduktion vordringen. Im CSP „EteRNA“ etwa wird den beteiligten Bürgerinnen und Bürger durch die Nutzung digitaler Infrastrukturen eine besonders vielfältige und weitgehende Inklusion versprochen, die von der wissenschaftlichen Zuarbeit über die Generierung wissenschaftlicher Erfindungen bis hin zur gemeinsamen Erstellung von Publikationen reichen kann. Beispielsweise zielt das von Wissenschaftlerinnen und Wissenschaftlern der Carnegie Mellon University sowie der Stanford University entwickelte und geleitete Projekt ${ }^{8}$ darauf ab, grundlegende Fragen zur RNA-Faltung zu beantworten. Nichtwissenschaftliche Akteurinnen und Akteure können „Puzzles“ rund um die Faltung von RNA-Molekülen lösen, indem sie aus den Bausteinen Adenin, Guanin, Cytosin und Uracil zunehmend komplexere RNA-Strukturen bilden. Durch das Spiel und die Beteiligung möglichst Vieler sollen kollektive Problemlösungsfähigkeiten genutzt werden, um Aufgaben zu bearbeiten, die sich einer effizienten algorithmischen Bearbeitung (zumindest bislang noch) entziehen.

Allerdings ist die unmittelbare Beteiligung an der Wissensproduktion durch technische Zugangshürden zunächst einmal ausgeschlossen. Nur wenige, besonders erfolgreiche und ausdauernde Spielerinnen und Spieler können in die Rolle eines Citizen Scientist schlüpfen, indem sie RNA-Designs im Spiel generieren, die dann in

\footnotetext{
5 https://eternagame.org/web/about/ [30.09.2019].

6 https://www.topothek.at/de/ [30.09.2019].

7 https://www.isi.fraunhofer.de/de/competence-center/neue-technologien/projekte/patient-science.html [30.09.2019].

8 https://en.wikipedia.org/wiki/EteRNA [30.09.2019].
} 
einem Stanford-Labor tatsächlich synthetisiert und geprüft werden. Die Zugangsregulierungen („Earn 10 more Tools to Start Real Experiments“ und „Unlock Lab Access to Help Invent Medicine“) werden jedoch erst sichtbar, sobald man sich als Nutzerin oder Nutzer registriert und anmeldet. Erst nach einer Registrierung und Anmeldung realisieren die Teilnehmerinnen und Teilnehmer allmählich, dass sie nicht bereits durch das unmittelbare Spielen in Prozesse der Wissensproduktion („Invent Medicine“) eingebunden sind, wie die Startseite in seiner Ansprache und dem großen grünen „Play Now“-Button suggeriert.

Die radikale Öffnung und spielerisch-massenhafte Einschließung prinzipiell Aller findet also im Rahmen eines Übungsmodus auf der Online-Plattform statt, die jedoch eine Beteiligung an der Wissensproduktion ausschließt. Der sozialen Öffnung von Forschung korrespondiert mithin eine Schließung gegenüber dem epistemischen Kern der Forschungstätigkeit. Daran ändern auch die gegenwärtigen Bestrebungen wenig, die etwa durch Zusammenschlüsse von beteiligten individuellen und organisationalen Akteuren, ${ }^{9}$ oder durch Definitionen, Leitlinien und Evaluationen darauf ausgerichtet sind, Aktivitäten auszuschließen, die den Ansprüchen an „gute“ oder „echte“ Citizen Science nicht genügen. Die wissenschaftspolitischen und zivilgesellschaftlichen Erwartungen nach einer zugleich gesellschaftlich legitimierten und epistemisch robusten Forschungs- und Innovationstätigkeit haben im Fall der Citizen Science ebenfalls ihren Preis: soziale Öffnung, jedoch weitgehende epistemische Geschlossenheit.

\section{4 ... und wo bleibt die Exzellenz?}

Natürlich ließen sich weitere Fälle anführen, die auf verschiedene Weisen versuchen, die großen Erwartungen an Forschung in Zeiten von Grand Challenges pragmatisch zu erfüllen. Da wären etwa die heute so gefeierten Reallabore: Aufbauend auf den Realexperimenten von Gross, Hoffmann-Riem und Krohn (2005) oszillieren sie zwischen den Modi der „Wissenserzeugung“ und „Wissensanwendung“ sowie „kontrollierten“ und „situationsspezifischen“ Randbedingungen (Schneidewind 2014). Reallabore schaffen Kontexte für Realexperimente, die dazu dienen, das Wissen über Transformationsprozesse zu verbessern - insbesondere indem sie die Produktion von denkund wünschbaren Alternativen vorantreiben. Doch genau die epistemischen Zwänge, die etwa mit der Standardisierung der Datenproduktion zusammenhängen, konterkarieren dieses Ziel. Reallabore liefern zwar robustere, da von heterogenen Akteuren produzierte Daten, aber kaum freie Exploration von Alternativen - und gerade dies sollte jedoch zur gesellschaftlichen Relevanz von Innovationen beitragen.

9 Etwa die European Citizen Science Association (ECSA, https://ecsa.citizen-science.net [30.09.2019]). 
Kurz: Forschung befindet sich derzeit im Gerangel widerstreitender Anforderungen - Innovation! und Relevanz! Nicht nur sind Maßnahmen Relevanzerzeugung im Innovationsprozess durch epistemische Kriterien der Forschungstätigkeit erkennbar eingeschränkt (etwa: Reproduzierbarkeit), und haben nicht selten eher ostentativen Charakter. Dies führt in aller Regel zu pragmatischen Lösungen: Hier wurden vor allem ein Trend zur Managerialisierung und zur sozialen Öffnung, jeweils mit speziellem Fokus auf Wissenschaftskommunikation hervorgehoben. Eine Demokratisierung der Wissenschaft im Sinne einer auch epistemisch erneuerten Wissenschaft (derzeit unter dem Stichwort RRI) ist momentan nur mit erheblichen Einschränkungen zu erkennen.

Darüber hinaus darf nicht vergessen werden, dass die Forderung nach Relevanz mit einer anderen Forderung nach wie vor konkurriert: derjenigen nach Exzellenz. Die „neue Wissensproduktion“ schafft immer neue Räume, Gelegenheiten und Verfahren, in denen heterogene Interessen artikuliert und ausgehandelt, Zielvereinbarungen getroffen sowie Ergebniskontrollen durchgeführt werden. Zunehmender ökonomischer und politischer Druck zeitigt dabei erhebliche, jedoch keineswegs einheitliche Effekte auf die Ziele und Formen wissenschaftlicher Wissensproduktion: Während die Einen auf Exzellenz setzen, favorisieren die Anderen partizipative Forschung. Beides geschieht, nota bene, im Namen der Qualität: Exzellenz argumentiert mit der Produktion international konkurrenzfähiger, Standort sichernder Innovation; partizipative Forschung hingegen auf die Produktion sozial robusten, relevanten und verantwortlichen Wissens. Dies beeinflusst auch die Formen der Wissensproduktion: Exzellenz setzt auf Synergie und Entsäulung der Forschung (Experten-Netzwerke), partizipative Forschung auf Kooperation mit außerwissenschaftlichen Stakeholdern (Netzwerke wechselseitigen Lernens). Beide Varianten vervielfältigen die Formen und Verfahren ihrer Qualitätssicherung: Während Exzellenz neben Peer Reviews auch Ratings und Rankings obligatorisch macht, ergänzt partizipative Forschung Peer Reviews etwa um Verfahren sozialer Validierung. Exzellenz und partizipative, relevanzorientierte Forschung repräsentieren mithin zwei distinkte Weisen, qualitativ herausragendes Wissen hervorzubringen. Die Gleichzeitigkeit beider Qualitätsdiskurse scheint ganz unterschiedliche gesellschaftliche Aufträge an wissenschaftliches Wissen $\mathrm{zu}$ indizieren: hier ökonomisch verwertbare Innovationskraft exzellenten Wissens, dort eher politisch verwertbare Legitimationskraft partizipativ generierten Wissens. Beide Formen indessen können als Übungen in der Erzeugung und kommunikativen Verstärkung legitimen Wissens und legitimierter Wissensproduktion gelesen werden.

Der neue Gesellschaftsvertrag mit der Wissenschaft scheint sich derzeit auf Innovation einzupendeln, entweder im Modus von Exzellenz oder im Modus von Relevanz. Alle Versuche, die Alternativen - Exzellenz (mit erhöhter Chance auf innerwissenschaftliche Anerkennung) oder Relevanz (mit erhöhter Chance auf gesellschaftlicher Anerkennung) - miteinander zu versöhnen, so das Fazit, ist zwar wissenschaftspolitisches Programm zur gegenwärtigen Legitimation der Forschung, stellt sie jedoch 
besonders in anwendungsnahen Feldern nicht selten vor Zerreißproben. Pragmatische Lösungen (Managerialisierung, nur-soziale Öffnung der Innovationstätigkeit) ebenso wie die Responsibilisierung von vorwiegend geistes- und sozialwissenschaftlichen Akteuren für verantwortliche Innovationsprozesse (Ethisierung der Forschung) sind derzeitige Antworten auf sie. Doch das wäre ein anderer Aufsatz. Ein Ende des Gerangels ist jedenfalls derzeit nicht in Sicht.

\section{Literatur}

Blättel-Mink, B. (2001). Wirtschaft und Umweltschutz: Grenzen der Integration von Ökonomie und Ökologie. Frankfurt/Main: Campus Verlag.

Böhme, G., W. van den Daele und W. Krohn (1973). Die Finalisierung der Wissenschaft. Zeitschrift für Soziologie 2(2), 128-144.

Böschen, S., M. Schneider und A. Lerf. Hrsg. (2004). Handeln trotz Nichtwissen: Vom Umgang mit Chaos und Risiko in Politik, Industrie und Wissenschaft. Frankfurt/Main, New York/NY: Campus Verlag.

Bröchler, S., G. Simonis und K. Sundermann. Hrsg. (1999). Handbuch Technikfolgenabschätzung. Berlin: edition sigma.

Bucchi, M. und B. Trench. Hrsg. (2008). Handbook of Public Communication of Science and Technology. London; New York/NY: Routledge.

Burkhart, C., M. Schmelzer und N. Treu. Hrsg. (2017). Degrowth in Bewegung(en): 32 alternative Wege zur sozial-ökologischen Transformation. München: Oekom.

Chilvers, J. (2013). Reflexive Engagement? Actors, Learning, and Reflexivity in Public Dialogue on Science and Technology. Science Communication 35(3), 283-310.

Collingridge, D. (1982). The Social Control of Technology. London: Pinter Publisher.

Cournand, A. F. und H. Zuckerman (1970). The Code of Science. Analysis and Some Reflections on its Future. Studium Generale 23(10), 941-962.

Dickel, S. (2017). Öffnung für alle: Einlösung oder Erosion des Projekts moderner Wissenschaft? TATUP. Zeitschrift für Technikfolgenabschätzung in Theorie und Praxis 26(1-2), 55-59.

Dickel, S. und M. Franzen (2016). Digitale Inklusion: Zur sozialen Öffnung des Wissenschaftssystems. Zeitschrift für Soziologie 44(5), 330-347.

Felt, U., M. Fochler und M. Strassnig (2011). Experimente partizipativer ELSA-Forschung. In: E. Grießler und H. Rohracher. Hrsg. Genomforschung - Politik - Gesellschaft: Perspektiven auf ethische, rechtliche und soziale Aspekte der Genomforschung. Wiesbaden: VS Verlag für Sozialwissenschaften, 33-67.

Föger, N. et al. (2016). RRI in Österreich. Positionspapier. Verantwortungsbewusste Forschung und Innovation. Begriffsbestimmung, Herausforderungen, Handlungsempfehlungen. Wien, Graz: Plattform RRI Österreich.

Fussler, C. (1996). Driving Eco-innovation: A Breakthrough Discipline for Innovation and Sustainability. London, Washington/DC: Pitman Publishing.

Gibbons, M. et al. (1994). The New Production of Knowledge: The Dynamics of Science and Research in Contemporary Societies. London: SAGE.

Godin, B. (2016). The Linear Model of Innovation: The Historical Construction of an Analytical Framework. Science, Technology, \& Human Values 31(6), 639-667.

Gregory, J. und S. J. Lock (2008). The Evolution of 'Public Understanding of Science': Public Engagement as a Tool of Science Policy in the UK. Sociology Compass 2(4), 1252-1265. 
Gross, M., H. Hoffmann-Riem und W. Krohn (2005). Realexperimente: Ökologische Gestaltungsprozesse in der Wissensgesellschaft. Bielefeld: Transcript.

Grunwald, A. (2011). Responsible Innovation: Bringing Together Technology Assessment, Applied Ethics, and STS Research. Enterprise and Work Innovation Studies 31, 10-19.

Guston, D. H. (2000). Retiring the Social Contract for Science. Issues in Science and Technology 16(4) [https://issues.org/p_guston/; 26.09.2019].

Hagendijk, R. und A. Irwin (2006). Public Deliberation and Governance: Engaging with Science and Technology in Contemporary Europe. Minerva 44(2), 167-184.

Hahn, J. und M. Ladikas (2014). Responsible Research and Innovation: A Global Perspective. Enterprise and Work Innovation Studies 10(10), 9-27.

Haklay, M. (2013). Citizen Science and Volunteered Geographic Information: Overview and Typology of Participation. In: D. Sui, S. Elwood und M. Goodchild. Hrsg. Crowdsourcing Geographic Knowledge: Volunteered Geographic Information (VGI) in Theory and Practice. Dordrecht: Springer Netherlands, 105-122.

Irwin, A. und B. Wynne. Hrsg. (1996). Misunderstanding Science?: The Public Reconstruction of Science and Technology. Cambridge, New York/NY: Cambridge University Press.

Knie, A. und D. Simon (2008). Evaluationen im Governance-Mix: Herausforderungen für das deutsche Wissenschaftssystem. Wissenschaftsmanagement (5), 24-29.

Lax, G. (2015). Das „lineare Modell der Innovation“ in Westdeutschland: Eine Geschichte der Hierarchiebildung von Grundlagen- und Anwendungsforschung nach 1945. Baden-Baden: Nomos.

Lindner, R. et al. (2016). Addressing Directionality: Orientation Failure and the Systems of Innovation Heuristic. Towards Reflexive Governance (Fraunhofer ISI Discussion Papers - Innovation Systems and Policy Analysis 52), 1-41 [http://hdl.handle.net/10419/145315; 26.09.2019].

Lindner, R. et al. (2016a). „Responsible Research and Innovation“als Ansatz für die Forschungs-, Technologie- und Innovationspolitik - Hintergründe und Entwicklungen (TAB-Hintergrundpapier 22) [https://www.tab-beim-bundestag.de/de/aktuelles/20161012.html; 26.09.2019].

Maasen, S. (2008). Exzellenz oder Transdisziplinarität: Zur Gleichzeitigkeit zweier Qualitätsdiskurse. In: S. Hornbostel, D. Simon und S. Heise. Hrsg. Exzellente Wissenschaft. Das Problem, der Diskurs, das Programm und die Folgen (IFQ-Working Paper 4). Bonn: Institut für Forschungsinformation und Qualitätssicherung, 23-32.

Maasen, S. (2010). Transdisziplinarität revisited - Dekonstruktion eines Programms zur Demokratisierung der Wissenschaft. In: A. Bogner, K. Kastenhofer und H. Torgersen. Hrsg. Inter- und Transdisziplinarität im Wandel? Neue Perspektiven auf problemorientierte Forschung und Politikberatung. Baden-Baden: Nomos, 247-268.

Maasen, S. und S. Dickel (2016). Partizipation, Responsivität, Nachhaltigkeit. In: D. Simon, A. Knie, S. Hornbostel und K. Zimmermann. Hrsg. Handbuch Wissenschaftspolitik. Wiesbaden: Springer, 1-18.

Nowotny, H., P. Scott und M. Gibbons (2001). Re-Thinking Science: Knowledge and the Public in an Age of Uncertainty. London: Polity.

Owen, R., P. Macnaghten und J. Stilgoe (2012). Responsible Research and Innovation: From Science in Society to Science for Society, with Society. Science and Public Policy 39(6), 751-760.

Özmen, E. (2015). Wissenschaft. Freiheit. Verantwortung: Über Ethik und Ethos der freien Wissenschaft und Forschung. Ordnung der Wissenschaft (2), 65-72.

Pansera, M. und R. Owen (2018). Innovation for De-Growth: A Case Study of Counter-hegemonic Practices from Kerala, India. Journal of Cleaner Production 197, 1872-1883.

Rammert, W. et al. Hrsg. (2016). Innovationsgesellschaft heute: Perspektiven, Felder und Fälle. Wiesbaden: VS Verlag für Sozialwissenschaften.

Schiederig, T., F. Tietze und C. Herstatt (2012). Green Innovation in Technology and Innovation Management: an Exploratory Literature Review. R\&D Management 42(2), 180-192. 
Schneidewind, U. (2014). Urbane Reallabore: Ein Blick in die aktuelle Forschungswerkstatt. Pnd / Online 3 [https://epub.wupperinst.org/frontdoor/index/index/year/2014/docld/5706; 26.09.2019].

Weingart, P. (1997). From „Finalization“ to „Mode 2“: Old Wine in New Bottles? Social Science Information 36(4), 591-613.

Weingart, P. (2005). Die Wissenschaft der Öffentlichkeit: Essays zum Verhältnis von Wissenschaft, Medien und Öffentlichkeit. Weilerswist: Velbrück Wissenschaft.

Weingart, P. (2011). Die Wissenschaft der Öffentlichkeit und die Öffentlichkeit der Wissenschaft. In: B. Hölscher und J. Suchanek. Hrsg. Wissenschaft und Hochschulbildung im Kontext von Wirtschaft und Medien. Wiesbaden: VS Verlag für Sozialwissenschaften, 45-61.

Weingart, P. et al. Hrsg. (2017). Perspektiven der Wissenschaftskommunikation im digitalen Zeitalter. Weilerswist: Velbrück.

Wieland, T. (2009). Neue Technik auf alten Pfaden? Forschungs- und Technologiepolitik in der Bonner Republik. Eine Studie zur Pfadabhängigkeit des technischen Fortschritts. Bielefeld: Transcript.

Yesley, M. (2008). What's ELSI got to do with it? Bioethics and the Human Genome Project. New Genetics and Society 27(1), 1-6. 